\title{
A room temperature 19-channel magnetic field mapping device for cardiac signals
}

\author{
G. Bison, ${ }^{1}$ N. Castagna, ${ }^{2}$ A. Hofer,${ }^{2}$ P. Knowles,${ }^{2}$ J.-L. Schenker, ${ }^{2}$ M. Kasprzak, ${ }^{2}$ H. Saudan, ${ }^{2}$ and A. Weis ${ }^{2}$, ${ }^{\circ}$ \\ ${ }^{1}$ Department of Neurology, Friedrich-Schiller-University, D-07740 Jena, Germany \\ ${ }^{2}$ Department of Physics, University of Fribourg, CH-1700, Fribourg, Switzerland
}

(Dated: October 22, 2018)

\begin{abstract}
We present a multichannel cardiac magnetic field imaging system built in Fribourg from optical double-resonance Cs vapor magnetometers. It consists of 25 individual sensors designed to record magnetic field maps of the beating human heart by simultaneous measurements on a grid of 19 points over the chest. The system is operated as an array of second order gradiometers using sophisticated digitally controlled feedback loops.
\end{abstract}

Signals derived from the electrophysiological processes in the human heart are important diagnostic tools for assessing cardiac disorder. For more than a century, measurements of body surface potentials (electrocardiography, ECG) generated by electrical currents within the myocardium have been a standard medical examination technique. Electrophysiological currents also generate a magnetic field, whose recording represents an alternative diagnostic tool [1], known as magnetocardiography (MCG). Three decades after the first recording of an MCG signal [2], Schneider et al. [3] in 1990 turned the MCG method into an imaging technique (cardiac magnetic field mapping, cMFM) by using a sensor array covering the whole chest. The magnetic signals (MCG) have a distinct advantage over the electrical signals (ECG) when considering source localization: the inverse problem of reconstructing the field sources from field maps is easier to solve in the magnetic case since the electrical conductivity of body tissue affects the transmission of magnetic signals to a much lesser degree than their electric counterpart. Modern cMFM systems [4] use superconducting quantum interference devices (SQUIDs) for recording the very weak magnetic field (peak amplitude $\approx 100 \mathrm{pT}$ ) generated by the heart. In the past years we have developed laser optical pumping magnetometers (LOPMs) [5] for biomagnetic applications [6]. The room temperature operation of LOPMs makes such magnetometers promising alternatives to SQUIDs by avoiding the expensive cryogenic cooling and the associated complex logistics in view of clinical applications. Since each LOPM sensor requires only a few $\mu \mathrm{W}$ of light power, a single laser can operate many dozens of individual sensor heads, a feature which has until now been unexploited. In this Letter we describe a cMFM system operating in Fribourg consisting of 25 individual LOPM sensors designed to record magnetic field maps of the beating human heart by simultaneous measurements on a grid of 19 points over the chest.

The LOPM sensors are based on optical-rf double resonance in the so-called $M_{x}$ configuration [5]. The sensor medium is a room temperature Cs vapor contained in a

*Corresponding author: antoine.weis@unifr.ch spherical paraffin-coated 7] $30 \mathrm{~mm}$ diameter glass bulb. Circularly polarized light from a diode laser, frequency locked to the $4 \rightarrow 3$ component of the cesium $D_{1}$ transition, creates a macroscopic magnetization in the vapor by optical pumping. The magnetization precesses around a static magnetic field $\vec{B}_{0}=B_{0} \hat{z}-$ nominally oriented at $\pi / 4$ with respect to the light propagation - with the Larmor frequency $\omega_{L}=\gamma\left|B_{0}\right|$, where $\gamma \approx 2 \pi \times 3.5 \mathrm{~Hz} / \mathrm{nT}$. The precession is coherently driven by a weak magnetic field $B_{1} \ll B_{0}$ oscillating at the frequency $\omega_{\mathrm{rf}}$. As a consequence, the light transmitted through the cell acquires an amplitude modulation at the frequency $\omega_{\mathrm{rf}}$. The modulation amplitude is resonantly enhanced at $\omega_{\mathrm{rf}}=\omega_{L}$ and the phase $\Delta \varphi$ between the drive and the response obeys (up to a constant phase offset)

$$
\Delta \varphi=\arctan \left(\frac{\omega_{L}-\omega_{\mathrm{rf}}}{\Gamma}\right),
$$

where the (HWHM) linewidth $\Gamma$ is typically $\approx 2 \pi \times 6 \mathrm{~Hz}$ [7] for our OPMs. Near resonance, $\Delta \varphi$ depends linearly on $B_{0}$ and on $\omega_{\text {rf }}$, so that feedback control of either $B_{0}$ or $\omega_{\text {rf }}$ can be used to keep the system on resonance.

The design of the 19-channel system was targeted at demonstrating the simultaneous operation of many LOPM sensors in close packing. Each sensor consists of a compact self-contained module including optical components to collimate and polarize the light, a Cs vapor cell, and a photodiode to detect the light transmitted through the cell. The components are mounted between two printed circuit boards (PCB) which carry coils for generating $B_{1}$. Each sensor module is placed in an additional pair of coils allowing the application of a small field, $B_{\mathrm{fb}}$, parallel to the field $B_{0}$. These feedback coils are realized as printed circuits on larger PCBs that serve as mechanical support structures positioning the sensors in horizontal planes (Fig. 11). An optimal sensor packing is achieved by arranging the modules on a hexagonal grid with a $50 \mathrm{~mm}$ spacing. A custom-made hologram splits the beam from the frequency-stabilized laser into 25 beams of equal intensity which are carried by multimode optical fibers to the individual sensor modules. The complete sensor system consists of 25 modules distributed in three planes that are vertically offset by $10 \mathrm{~cm}$ (Fig. 11). The lower plane, closest to the subject's chest, is equipped with 19 primary mapping modules. The mid- 


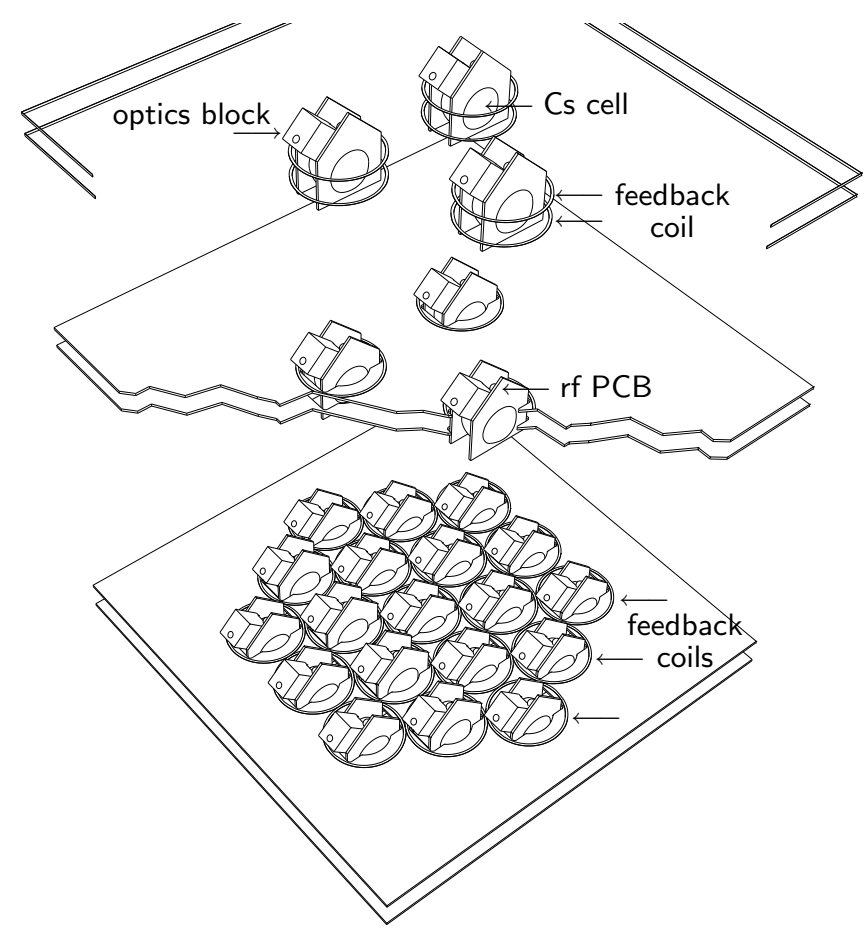

Figure 1: Perspective drawing of the 25-sensor 19-channel cMFM system installed in our Al shielding room in Fribourg. The bottom plane holds the 19 measurement channels, while the mid plane and top plane hold three sensors each, used for field and gradient fluctuation suppression.

dle and upper planes each carry three sensors providing reference signals for stabilizing $B_{0}$ and its linear gradient, respectively. The sensor system is mounted inside of a two layer $\mathrm{Al}$ shielding room that reduces ambient magnetic field fluctuation which are dominated by $50 \mathrm{~Hz}$ (power line) and $162 / 3 \mathrm{~Hz}$ (railway) interference. The person to be diagnosed is placed underneath the sensor system on a nonmagnetic bed. Three pairs of large coils surrounding the shielding room are used to create a vertical field $B_{0} \approx 9 \mu \mathrm{T}$ (corresponding to $\omega_{L}=2 \pi \times 31.5 \mathrm{kHz}$ ) at the sensor location. Transverse $d B_{0} / d x, d B_{0} / d y$ gradients are compensated using additional large coils and constant current supplies.

The control electronics is implemented in a digital FPGA board which digitizes, via ADCs, the photocurrent from each module after transimpedance amplification, and which generates, via DACs, the currents driving the rf coils and the feedback currents for the sensors in the lower and upper planes. For each sensor the phase difference $\Delta \varphi$ is determined using a numerical lock-in detection algorithm. The near resonant linear dependence of the phase signals form suitable error signals that are used, after proportional-integral-differential (PID) amplification, as feedback signals in the various servo-loops discussed below. The middle plane carries three sensors that serve as reference sensors which track changes of the DC offset field $B_{0}$ (applied field plus external field changes). In each of the three middle plane sensors, $\Delta \varphi$ is actively locked to zero by servo adjustments of the corresponding three drive frequencies $\omega_{\text {rf }}$ generated by a numerically controlled oscillator. The computed average of those three individual frequencies or, alternatively, the frequency of a single (centered) sensor is then used as common drive frequency $\omega_{\text {com }}$ for the sensors in the top and bottom planes. In both ways the drive frequency tracks fluctuations and drifts of $B_{0}$.

When using a common drive frequency $\omega_{\text {com }}$, the signals $\Delta \varphi$ of the sensors in the top and bottom planes are proportional to the difference between the field at their locations and the field in the middle plane. These sensors thus form first-order hardware gradiometers. In particular, the signal $\Delta \varphi$ of the top sensors measures the linear vertical magnetic field gradient, while being almost insensitive to the cardiac field. The phase differences $\Delta \varphi$ of the upper sensors are locked to zero via servo loops injecting current into each of their respective feedback coils. In this way the local field at each upper sensor location follows the field in the middle plane. When simultaneously injecting currents of the same magnitude, but of opposite polarity, into the bottom layer feedback coils, the linear vertical field gradient along the vertical extension of the sensor system, and its fluctuations are actively compensated. Since the number of upper reference sensors is less than the number of imaging sensors in the bottom plane, only the average of the upper correction currents is used as feedback for the lower plane.

When the average field and the linear vertical gradient fluctuations are suppressed by the feedback loops described above, the remaining phase differences $\Delta \varphi$ of the lower plane sensors are proportional to the second order gradient of $B_{0}$. However, those phase differences are not directly measured: by using yet another set of servoloops driving the lower plane feedback coils, the 19 phase differences $\Delta \varphi$ are removed, forcing all sensors to be in resonance with $\omega_{\text {com. }}$. The 19 time-dependent feedback currents of the latter loop thus consist of the biomagnetic signals of interest and fluctuations of $B_{0}$ 's second order gradient. These signals are recorded at a sampling rate of $5 \mathrm{kHz}$. This magnetic feedback scheme relying on three distinct sets of servo-loops is of general interest for any gradiometric arrangement of multiple, closely packed magnetometers. It may be particularly useful for coherent population trapping magnetometers (CPTM), which have been used for biomagnetic field detection [8] but have not yet been reported to be scalable to many channels. Close-packed CPTMs can be operated in an all-optical mode by using one optical modulator per channel, however, magnetic feedback is probably a more cost effective operation method for a multichannel CPTM implementation. The second order gradiometer scheme reduces field fluctuations by a factor of $>1000$ to a level of $900 \mathrm{fT} / \sqrt{\mathrm{Hz}}$ at $0.1 \mathrm{~Hz}$. The noise of the gradiometer signal drops towards larger frequencies and reaches 300 $\mathrm{fT} / \sqrt{\mathrm{Hz}}$ at $100 \mathrm{~Hz}$.

Since the sensors are closely packed, the $B_{\mathrm{fb}}$ field of a given feedback coil induces crosstalk with its neighbours, 


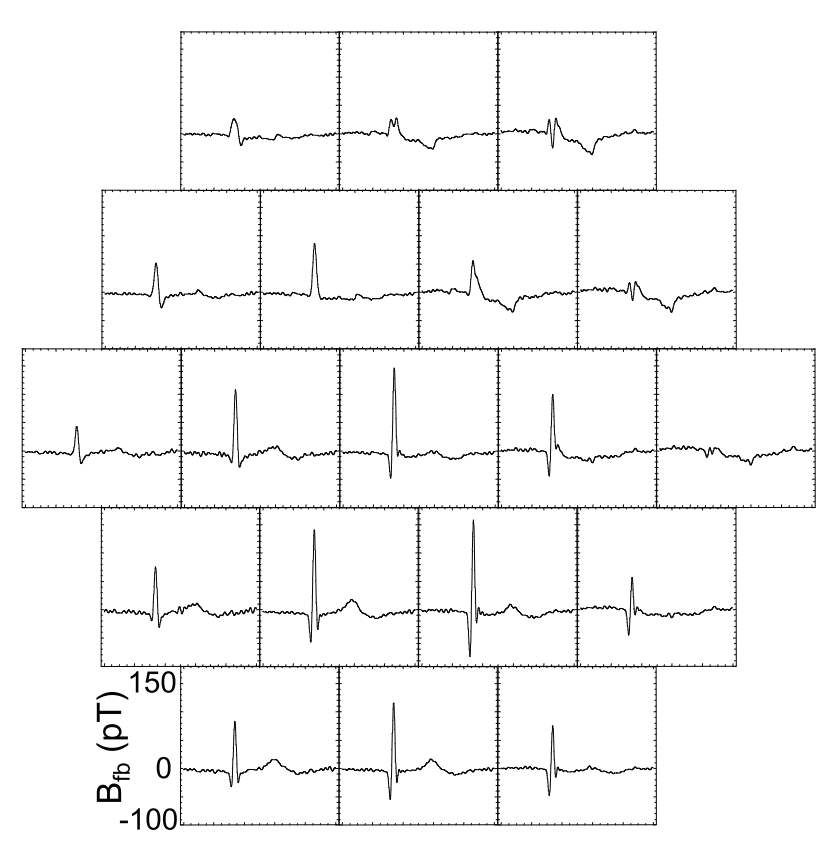

Figure 2: Time series showing (averaged) MCG traces of a healthy subject for each of the 19 sensor positions. Each trace is $1 \mathrm{~s}$ long and all graphs have the same (280 pT) vertical extension. For this measurement only a single (centered) reference sensor was used in the middle plane.
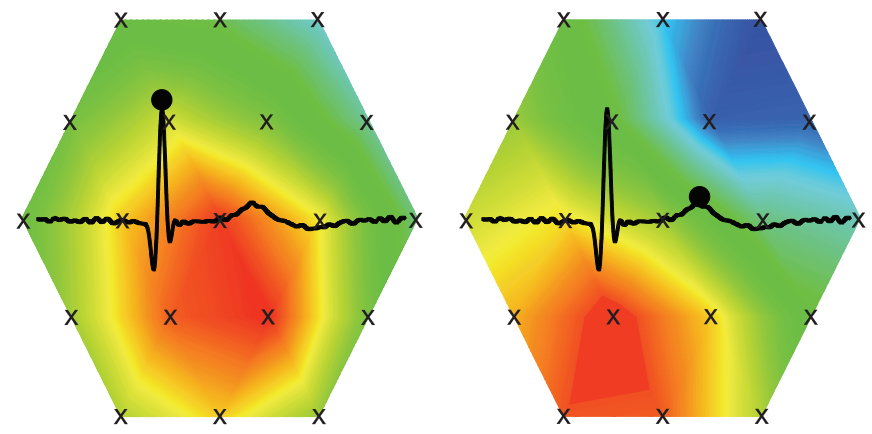

Figure 3: (Color online) Magnetic field map for the out-ofchest component of the cardiac field. Crosses show sensor positions, and the superimposed trace (bottom center trace from Fig. 2) identifies, via the black dot, the time for which the map was evaluated. Left (right): maps at the R-peak (T-wave). although the effect is small enough $(<10 \%)$ that the system operates without online corrections. The measured crosstalk $d B_{i} / d I_{j}$ (field in sensor $i$ induced by current in feedback coil $j$ ) can be used for offline data correction $[9]$.

Figure 2 shows an example of 19 simultaneously recorded MCG traces obtained by averaging signals from 66 heartbeats. The data represents the second order gradient of the out-of-chest component of the cardiac field. A 3-lead ECG signal served as timing reference for aligning the individual heart beats when averaging. The total recording time is less than two minutes, a huge improvement compared to the two hours required when stepping a single sensor over the chest [6]. The signals are (lowpass) filtered offline by multiplying their Fourier spectra with a smooth function passing from 1 to 0 in the band of 40 to $50 \mathrm{~Hz}$. Systematic implementation of crosstalk correction is a work in progress and thus has not been applied to the shown data. The residual oscillations are due to railway interference $(162 / 3 \mathrm{~Hz})$. In Fig. 3. magnetic field intensity maps for two given times (R-peak, T-wave) of the cardiac cycle are shown. The smooth pattern is obtained by a numerical interpolation between the discrete measurement points (shown as crosses).

We have reported the operation of a 19 channel secondorder gradiometer system based on atomic magnetometers for MCG measurements, capable of recording a magnetic field map in about two minutes in a weakly shielded environment. The $19+3+3$ system is now being used at the University of Fribourg for an initial series of measurements on both volunteers and cardiac patients. A $57+13+13$ channel system is currently being built at the University Hospital in Jena (Germany). Tests of that system in a clinical environment should start early in 2010 .

\section{Acknowledgments}

This work was supported by the Velux Foundation, the Swiss National Science Foundation (\#200020-119820), and the German Federal Ministry for Education and Research (\#0315024 B). The authors thank Dr. S. N. Erné of BMDSys GmbH for help in the sensor layout design.
[1] W. Andrä and H. Nowak, eds., Magnetism in Medicine (Wiley-VCH Verlag GmbH, Berlin, 1998), ISBN 3-52740221-7.

[2] G. M. Baule and R. McFee, Am. Heart J. 66, 95 (1963).

[3] S. Schneider, E. Hoenig, H. Reichenberger, K. AbrahamFuchs, W. Moshage, W. Oppelt, A. Stefan, A. Weikl, and A. Wirth, Radiology 176, 825 (1990).

[4] BMDSys GmbH, http://www.bmdsys.com

[5] S. Groeger, G. Bison, J.-L. Schenker, R. Wynands, and A. Weis, Eur. Phys. J. D 38, 239 (2006).

[6] G. Bison, R. Wynands, and A. Weis, Opt. Expr. 11, 904
(2003).

[7] N. Castagna, G. Bison, G. Di Domenico, A. Hofer, P. Knowles, C. Macchione, H. Saudan, and A. Weis, Appl. Phys. B 96, 763 (2009).

[8] J. Belfi, G. Bevilacqua, V. Biancalana, S. Cartaleva, Y. Dancheva, and L. Moi, J. Opt. Soc. Am. B 24, 2357 (2007).

[9] A. Hofer, G. Bison, N. Castagna, P. Knowles, J.-L. Schenker, and A. Weis, unpublished (2009). 